\title{
Status on the Michigan-MIT Ultra-Cold Polarized Hydrogen Jet Target
}

\author{
V. G. Luppov, B. B. Blinov, J. A. Bywater, S. Chin, V. V. Churakov*, \\ G. R. Court ${ }^{\dagger}$, W. A. Kaufman, D. Kleppner ${ }^{\ddagger}$, A. D. Krisch, Yu. M. Melnik*, \\ J. B. Muldavin, T. S. Nurushev, J. S. Price, A. F. Prudkoglyad*, \\ R. S. Raymond, V. B. Shutov**, J. A. Stewart \\ Randall Lab of Physics, University of Michigan, Ann Arbor, 48109-1120, USA \\ *Inst. for High Energy Physics, RU-142284, Protvino, Russia \\ †Physics Department, Liverpool University, P.O. Box 142, Liverpool, L693BX, Great Britain \\ ${ }^{\ddagger}$ Department of Physics, Massachusets Institute of Technology, Cambridge, \\ Massachusetts 02199, US.4 \\ ** Joint Institute for Nuclear Research, RU-141980, Dubna, Russia
}

\begin{abstract}
Progress on the Mark-II ultra-cold polarized atomic hydrogen gas Jet target for the experiments NEPTUN-A and NEPTUN at UNK is presented. We describe the performance and the present status of different components of the jet.
\end{abstract}

To study of spin effects in high energy p-p collisions in the NEPTUN-A (1) and NEPTUN (2) experiments we are developing an ultra-cold high density jet target of proton-spin polarized hydrogen atoms. This method uses an ultracold separation cell coated with superfluid helium- 4 and a high magnetic field to produce an electron-spin polarized atomic hydrogen beam $(3,4)$.

The Michigan ultra-cold prototype Jet (4) produced an electron-spin polarized atomic hydrogen beam with dc flow of $3.7 \cdot 10^{15} \mathrm{H} / \mathrm{s}$, which corresponds to a density of $3 \cdot 10^{11} \mathrm{H} / \mathrm{cm}^{3}$. With our Mark-II Jet we expect to reach nuclearspin polarized hydrogen atom density of about $5 \cdot 10^{12} \mathrm{H} / \mathrm{cm}^{3}$, corresponding to the target thickness of $10^{13} \mathrm{H} / \mathrm{cm}^{2}$. We plan to have a $2 \mathrm{~cm}$ jet width along the accelerator beam.

A schematic diagram of the Mark-II Jet is shown in Figure 1. The atomic hydrogen is produced in a room temperature rf dissociator and guided to an ultra-cold stabilization cell coated with superfluid helium- 4 . The double walls of the cell form the mixing chamber of the dilution refrigerator. The cell's entrance and exit apertures are respectively located at about $95 \%$ and $60 \%$ of the central field of the $12 \mathrm{~T}$ superconducting solenoid. After the hydrogen atoms are thermalized by collisions with the cell surface, the magnetic field gradient physically separates the atoms according to their electron-spin state. The atoms in the two lowest hyperfine states $(|3>| 4\rangle$,$) are attracted toward$ the high field region and escape from the cell. They recombine on bare surfaces and are cryopumped. The atoms in the two higher hyperfine states $(|1>| 2>$, are repelled toward the low field region and effuse from the exit aperture, forming the electon-spin polarized beam. 


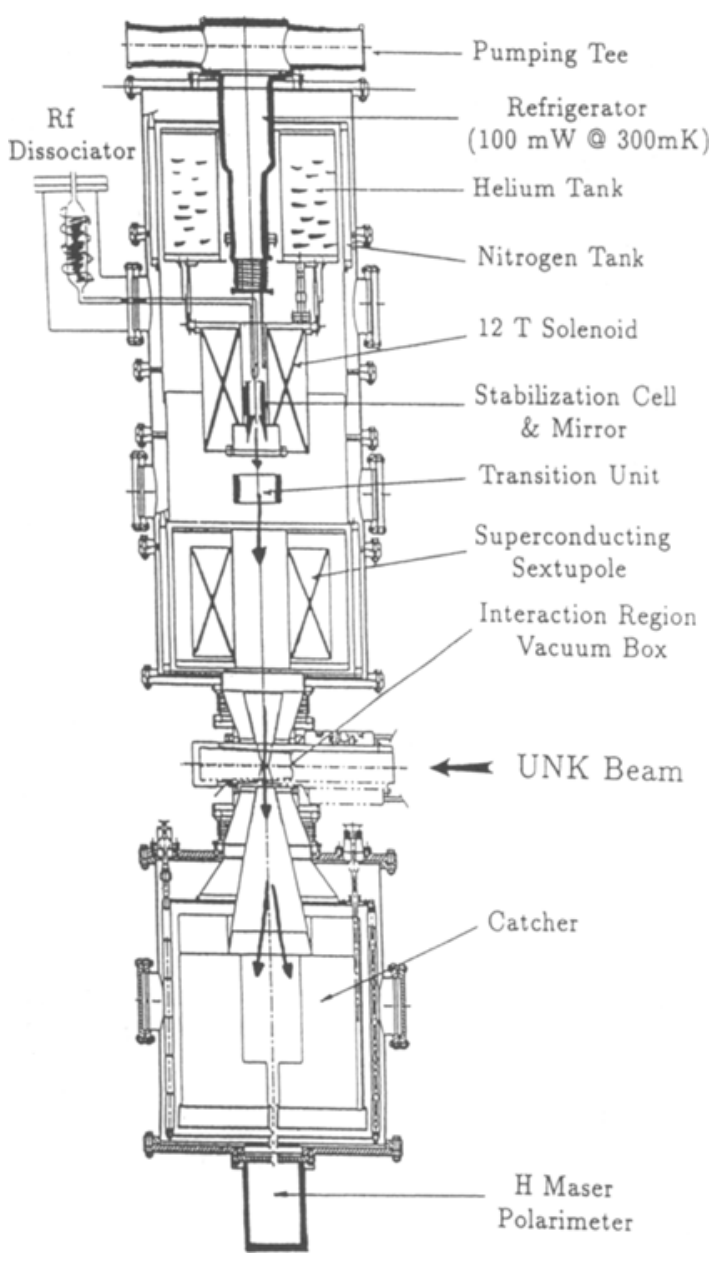

FIGURE 1. Schematic diagram of the Mark-II Ultra-Cold Jet.

After an rf transition unit, which interchanges atoms in states $|2\rangle$ and $|4\rangle$ we have a superconducting sextupole. The sextupole selects atoms in electron spin state $+1 / 2$ by focusing atoms in state $\mid 1>$ into the interaction region and defocusing atoms in state $|4\rangle$, which are then cryopumped. The nuclearspin polarized beam that passes through the interaction region is caught by a cryopumping catcher. A maser polarimeter below the catcher monitors the beam proton polarization.

Most of the vacuum jackets, nitrogen tanks and the main helium reservoir have been built and tested. The $12 \mathrm{~T}$ solenoid has been wound and successfully tested. It consists of an inner $\mathrm{Nb}_{3} \mathrm{Sn}$, outer $\mathrm{NbTi}$ coils and a $\mathrm{NbTi}$ bucking coil. Due to the bucking coil, located downstream of the solenoid the axial field falls off with about a $1 \mathrm{~T} / \mathrm{cm}$ gradient and has a very short tail, which lightens the rf unit magnetic shielding problem. 


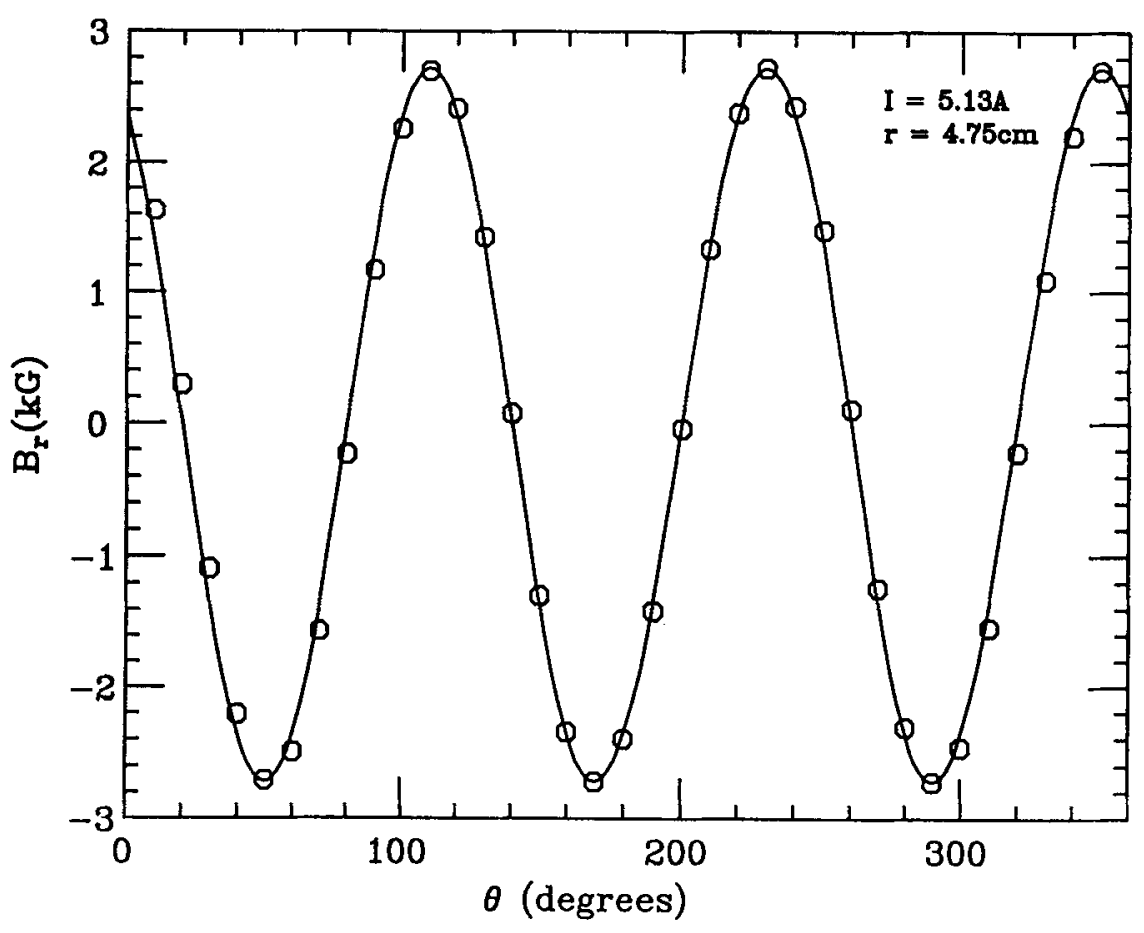

FIGURE 2. Azimuthal scan of the sextupole radial magnetic field near pole tips. o measurements, solid line - sinusoidal fit to the data.

The $100 \mathrm{~mW}$ dilution refrigerator has been built and is now undergoing cryogenic tests. It is designed to operate at $300 \mathrm{mK}$ and circulate $30 \mathrm{mmol} / \mathrm{s}$ of helium-3.

We plan to use a helium-film coated quasi-parabolic mirror to better focus the atomic hydrogen effusing from the stabilization cell (5). Simulations indicate that with no mirror approximately $25 \%$ of the beam can be focused to the $10 \mathrm{~mm} \times 20 \mathrm{~mm}$ interaction spot. We expect that a specially designed mirrow will significantly increase that fraction. We are now working on a mirror shape taking into account the axial and radial gradients of the solenoid field.

The $\mid 2>$ to $\mid 4>$ rf unit uses the adiabatic passage transition method. A ring dielectric resonator is used to accomodate the $6 \mathrm{~cm}$ beam diameter. The preliminary design and some tests on a prototype unit have been done (6).

The focusing sextupole magnet with superconducting coils and iron poles has been built and tested. To increase the Mark-II acceptance the $20 \mathrm{~cm}$ long sextupole has a bore diameter of $11 \mathrm{~cm}$. Simulations tailored to the Mark II 
geometry show that an optimum pole tip magnetic field is about $3.2 \mathrm{kG}$. This number corresponds to a coil current of $6 \mathrm{~A}$. One of the sextupole test results is shown in Figure 2.

The catcher consists of $13 \mathrm{~m}^{2}$ of copper cryocondensation fins cooled to $3 \mathrm{~K}$. It will keep the background pressure in the interaction region chamber at a level of $10^{-9}$ Torr. All parts of the catcher, except the upper shielding, have been fabricated and are now under assembly.

The MIT hydrogen maser polarimeter has been built and is now under test at Michigan. To monitor the state populations it employs a room temperature hydrogen maser that is operated in a transient, sub-threshold mode (7).

\section{ACKNOWLEDGEMENTS}

This work is supported by the U.S. Department of Energy.

\section{REFERENCES}

1. Krisch, A. D., "Polarization in High $P_{\perp}^{2} p-p$ Elastic Scattering at UNK" in Proceedings of the Workshop on Physics at UNK, Protvino, Russia, 1989, pp. 152-174.

2. Solovianov,V. L., "Study of Spin Effects with Jet Target on the UNK Internal Beam Facility at 0.4-3.0 TeV/c"in Proceedings of the Workshop on the Experimental Program at UNK, Protvino, Russia, 1987, pp. $191-213$

3. Mertig, M., et al., "Accumulation of Hydrogen Atoms in a Low Temperature Storage Cell of a Polarized Hydrogen Gas Jet Source "in Proceedings of the 9-th Intern. Symposium on High Energy Physics, Bonn, 1990, pp. 164-167.

4. Kaufman, W. A. , Roser, T., and Vuaridel, B. , Nuclear Instruments and Methods A 335, 17-29 (1993).

5. Luppov, V. G. , et al., Physical Review Letters 71, 2045-2048 (1993).

6. Muldavin, J. B., and Price, J. S., "Progress in Development of a $4 \mathrm{~K}$ RF Transition Unit for Use with an Ultra-Cold Polarized Hydrogen Beam", in Bulletin of the American Physical Society, Vol.39, No. 2, p.1109 (1994)

7. Kleppner, D., Goldenberg, H. M., and Ramsey, N. F., Physical Review 126, 603-615 (1962) 